\title{
Diagnostic Value of Matrix Metalloproteinase-9 and Tissue Inhibitor of Matrix Metalloproteinase-1 in Sepsis-Associated Acute Kidney Injury
}

\author{
Suzana Bojic, ${ }^{1}$ Jelena Kotur-Stevuljevic, ${ }^{2}$ Nevena Kalezic, ${ }^{3}$ Predrag Stevanovic, ${ }^{3}$ \\ Zorana Jelic-Ivanovic, ${ }^{2}$ Dragoljub Bilanovic, ${ }^{3,4}$ Lidija Memon, ${ }^{5}$ \\ Mladen Damnjanovic, ${ }^{6}$ Zdravko Kalaba $^{1}$ and Sanja Simic-Ogrizovic ${ }^{3}$ \\ ${ }^{1}$ Department of Anaesthesiology, Resuscitation and Intensive Care, Clinical Hospital Center Bezanijska Kosa, \\ Belgrade, Serbia \\ ${ }^{2}$ Faculty of Pharmacy, University of Belgrade, Belgrade, Serbia \\ ${ }^{3}$ School of Medicine, University of Belgrade, Belgrade, Serbia \\ ${ }^{4}$ Department of Surgery, Clinical Hospital Center Bezanijska Kosa, Belgrade, Serbia \\ ${ }^{5}$ Clinical Chemistry Laboratory, Clinical Hospital Center Bezanijska Kosa, Belgrade, Serbia \\ ${ }^{6}$ General Hospital Stefan Visoki, Smederevska Palanka, Serbia
}

Sepsis-associated acute kidney injury (SA-AKI) severely impacts morbidity and mortality in surgical patients with sepsis. Matrix metalloproteinase-9 (MMP-9) and tissue inhibitor of matrix metalloproteinase-1 (TIMP-1) have an important role in pathophysiology of sepsis but they have been unexplored in SA-AKI. We aimed to investigate the role of MMP-9 and TIMP-1 in septic surgical patients with SA-AKI and to evaluate them as diagnostic biomarkers of SA-AKI. This prospective observational study compared 53 major abdominal surgery patients with sepsis divided into SA-AKI $(n=37)$ and non-SA-AKI $(n=16)$ group to 50 controls without sepsis matched by age, gender, comorbidities and type of surgery. Blood and urine samples from septic patients were collected on admission to ICU and 24, 48, 72 and $96 \mathrm{~h}$ later and once from the controls. The levels of MMP-9, TIMP-1, neutrophil gelatinase-associated lipocalin (NGAL), kidney injury molecule-1, urea and creatinine were measured. MMP-9/TIMP-1 ratio and disease severity scores, such as Sequential Organ Failure Assessment (SOFA), were calculated. Septic patients with SA-AKI had higher serum TIMP-1 levels and lower serum MMP-9 levels and lower MMP-9/TIMP ratio, compared to septic patients without SA-AKI and controls. The levels of these biomarkers did not change significantly over time. MMP-9, TIMP-1 and MMP-9/TIMP-1 ratio correlated with urea, creatinine, NGAL, and SOFA scores. Moreover, using the area under ROC curve, we showed that TIMP-1 and MMP-9/TIMP-1 ratio, but not MMP-9, were good diagnostic biomarkers of SA-AKI. We report for the first time the potential diagnostic value of TIMP-1 and MMP-9/TIMP-1 ratio in SA-AKI.

Keywords: acute kidney injury; biomarker; matrix metalloproteinase-9; sepsis; tissue inhibitor of matrix metalloproteinase-1

Tohoku J. Exp. Med., 2015 October, 237 (2), 103-109. (C) 2015 Tohoku University Medical Press

\section{Introduction}

Acute kidney injury (AKI) is associated with a significant increase in mortality, duration of hospitalization and costs across a broad spectrum of conditions (Chertow et al. 2005). Sepsis is the foremost precipitant of AKI (Khwaja 2012). Sepsis-associated AKI (SA-AKI) is even considered as a distinct subtype of AKI due to its unique pathophysiology (Alobaidi et al. 2015). AKI is also a serious complication in surgical patients with major abdominal surgery and sepsis as some of the most important risk factors (Kheterpal et al. 2009; Teixeira et al. 2014).

Early diagnosis of AKI is generally accepted as a goal of paramount importance since it can improve the disease outcome (Khwaja 2012). Current diagnostic criteria for AKI, serum creatinine levels and diuresis, have serious limitations (Khwaja 2012). Diagnostic value of certain novel biomarkers of AKI, neutrophil gelatinase-associated lipocalin (NGAL) and kidney injury molecule-1 (KIM-1), is evident in cardiac surgery (Shao et al. 2014; Zhou et al. 2015) and sepsis (Tu et al. 2014; Dai et al. 2015) but not so much in abdominal surgery (Shavit et al. 2011; Cullen et al.

Received February 12, 2015; revised and accepted August 27, 2015. Published online September 19, 2015; doi: 10.1620/tjem.237.103. Correspondence: Suzana Bojic, M.D., Department of Anaesthesiology, Resuscitation and Intensive Care, Clinical Hospital Center Bezanijska Kosa, Bulevar Mihajla Pupina 163/13, 11000 Belgrade, Serbia.

e-mail: subojic@yahoo.com 
2014). The quest for new diagnostic biomarkers of AKI remains ongoing.

Matrix metalloproteinase-9 (MMP-9) is a multidomain zinc metalloproteinase released by inflammatory cells. Aside from the extracellular matrix remodeling, MMP-9 regulates the activity of numerous cytokines, chemokines, receptors, growth factors and cell adhesion molecules essential to inflammation (Vandooren et al. 2013; Galliera et al. 2015). The activity of the MMP-9 is closely regulated on multiple levels including the inhibition by regulatory proteins, such as tissue inhibitor of matrix metalloproteinase-1 (TIMP-1) (Galliera et al. 2015). Surgery-induced inflammation (Helmersson-Karlqvist et al. 2012; Lindholm et al. 2015) and sepsis (Muhl et al. 2011; Lauhio et al. 2011; Yazdan-Ashoori et al. 2011; Lorente et al. 2014; Martin et al. 2014; Wang et al. 2014) are associated with altered levels of MMP-9 and TIMP-1. Increased MMP-9 and TIMP-1 expression has also been reported in a variety of AKI animal model studies (Maitra et al. 2010; Teng et al. 2012) but only one clinical study evaluated MMP-9 as diagnostic biomarker of AKI and not SA-AKI per se (Han et al. 2008). So far, clinical data on MMP-9 and TIMP-1 levels in SA-AKI have been lacking.

In the present study, we focus on the septic patients who underwent major abdominal surgery, because they are considered as high risk patients for developing AKI. The aim of our study was to investigate the serum levels of MMP-9 and TIMP-1 and the MMP-9/TIMP-1 ratio in major abdominal surgery patients with SA-AKI and to evaluate them as potential diagnostic biomarkers of SA-AKI.

\section{Methods}

This prospective, observational study enrolled adult patients admitted to university hospital surgical intensive care unit (ICU) during 2012 and 2013. Institutional Ethical Committee of Clinical Hospital Center Bezanijska Kosa, Belgrade, Serbia approved this study. A written informed consent was obtained from participants or their legal representatives.

\section{Patients}

The study included 53 major abdominal surgery patients with sepsis and 50 control patients without sepsis matched by age, gender, comorbidities and a type of surgery. The patients with sepsis were enrolled if they were $>18$ years of age, if they were admitted to ICU for the treatment of sepsis and had at least one major abdominal surgery during this hospitalization. The septic patients were then observed for occurrence of AKI during their entire ICU stay. If AKI was diagnosed, patients were allocated to SA-AKI group. Consequentially, if AKI was not diagnosed, patients were allocated to sepsis without SA-AKI group. The control group patients were enrolled if they were $>18$ years of age, if they were routinely admitted to ICU after major abdominal surgery and whose recovery was uneventful. Major abdominal surgery was defined as a procedure in which peritoneum is entered, organs are removed or normal anatomy is altered. The exclusion criteria were age $<18$ years, history of chronic kidney disease, end-stage organ disease, chemotherapy and radiotherapy in the past 30 days, immunosuppressant therapy, major trauma, burns, cardiogenic or hemorrhagic shock and participation in another clinical trial during this hospitalization. All patients received standard routine care.

\section{Clinical assessment}

Sepsis was diagnosed according to American College of Chest Physicians/Society of Critical Care Medicine Consensus Conference criteria (Bone et al. 1992). The primary outcome of this study was the development of AKI during the ICU stay. AKI was diagnosed based on Kidney Disease/Improving Global Outcomes (KDIGO) criteria (Khwaja 2012). In order to quantify comorbidities, American Society of Anesthesiologists (ASA) physical status assessment (Little 1995) was performed. In the septic patients, only comorbidities, which were present prior to sepsis, were taken into consideration and all changes in physical status caused by sepsis were disregarded. In the control group, ASA physical status was routinely assessed prior to the surgery. Disease severity scores, Acute Physiology and Chronic Health Evaluation II (APACHE II) (Berger et al. 1992) and Sequential Organ Failure Assessment (SOFA) (Moreno et al. 1999), were calculated daily, at the time of sample collection. Modification of Diet in Renal Disease (MDRD) formula was used to calculate glomerular filtration rate (GFR) (Levey et al. 1999).

\section{Blood and urine assays}

Blood and urine samples from septic patients were collected during the first hour following the admission to ICU $(0 \mathrm{~h})$ and $24 \mathrm{~h}$, $48 \mathrm{~h}, 72 \mathrm{~h}$ and $96 \mathrm{~h}$ later. Blood and urine samples from the control group were collected once, in the morning after the surgery. Blood samples were drawn into standard collection tubes with a cloth activator. Urine samples were collected from urinary catheter using a standard urine collection technique. Centrifuged serum and urine aliquots were then stored to $-80^{\circ} \mathrm{C}$ until further analysis. MMP-9 and TIMP-1 concentrations were measured by enzyme-linked immunosorbent assay (ELISA) kits (Research and Diagnostics Systems, Minneapolis, MN, USA). MMP-9/TIMP-1 ratio was calculated as follows: MMP-9/TIMP-1 ratio = MMP-9 (ng/ml)/TIMP-1 (ng/ $\mathrm{ml}$ ). Novel markers of renal function, serum and urine NGAL and urine KIM-1, were also measured using ELISA kits (ABBOTT Diagnostics, IL, USA and R\&D Systems Inc., MN, USA respectively). Standard markers of renal function, serum urea and creatinine were measured employing routine laboratory methods on the day of the collection.

\section{Statistical analysis}

Statistical analysis was performed in SPSS 15.0 software (SPSS Inc., Chicago, IL, USA). We did not perform any power analysis due to missing pre-study data. The sample of 53 septic patients was assessed to be adequate based on previous MMP-9 and TIMP1studies in other septic patient populations (Muhl et al. 2011; YazdanAshoori et al. 2011; Martin et al. 2014). The normality of data was assessed with Kolmogorov-Smirnov test. The data are presented as median and $25^{\text {th }}$ to $75^{\text {th }}$ quartile or frequencies. The within-group variations were analyzed using the Friedman's test. The differences between the groups were compared using the Mann-Whitney U or Chi square test, where appropriate. To test possible correlations between variables, Kendall tau-b test was employed. Areas under receiver operating characteristic curves (AUC-ROC) for diagnosis of AKI were calculated using $0 \mathrm{~h}$ and mean values of tested biomarkers 
as independent variables. The significance level for all statistical tests was set at 0.05 .

\section{Results}

Baseline demographic and clinical characteristics of septic patients and the control group are summarized in Table 1. Thirty-seven of 53 septic patients $(69.8 \%)$ and none of the controls were diagnosed with SA-AKI. We did not observe significant differences in age, gender, comorbidities, expressed as ASA physical status, or type of surgery between patients groups. As expected, disease severity scores were higher in the septic patients with SA-AKI than in the septic patients without SA-AKI and the control group as well as in septic patients without SA-AKI compared to control group.

Table 2 presents baseline laboratory parameters in the septic patients and the control group. Expectedly, the septic patients with SA-AKI had significantly higher levels of urea, creatinine, NGAL, and KIM-1 but lower GFR relative to the septic patients without SA-AKI and the control group. The septic patients with SA-AKI also had significantly higher TIMP-1 levels and lower MMP-9/TIMP-1 ratio compared to the septic patients without SA-AKI and the control group. We observed lower levels of MMP-9 in the septic patients with SA-AKI relative to the septic patients without SA-AKI and the control group, but this difference did not reach statistical significance. The septic patients without SA-AKI also had higher TIMP-1 levels compared to the control group.

Fig. 1 depicts the dynamics of MMP-9, TIMP-1 and MMP-9/TIMP-1 ratio in the septic patients with and without SA-AKI with comparison to the control group. The septic patients with SA-AKI had lower MMP-9 levels than the septic patients without SA-AKI at $72 \mathrm{~h}$ and $96 \mathrm{~h}$ follow-

Table 1. Baseline demographic and clinical characteristics of patients with sepsis and the control group.

\begin{tabular}{llcc}
\hline & \multicolumn{1}{c}{ Control group } & Sepsis without SA-AKI & Sepsis with SA-AKI \\
\hline No of patients & 50 & 16 & 37 \\
Age (years) & $65.0[57.75-74.0]$ & $70.00[57.5-77.25]$ & $70.00[61.5-75.0]$ \\
Gender $(\mathrm{m} / \mathrm{f})$ & $22 / 28$ & $9 / 7$ & $19 / 18$ \\
ASA $1 / 2 / 3 / 4 / 5$ & $0 / 50 / 0 / 0 / 0$ & $2 / 12 / 2 / 0 / 0$ & $0 / 35 / 2 / 0 / 0$ \\
APACHE II & $7[5-7.5]$ & $16[13.5-18]^{\mathrm{a}}$ & $25[18-30.75]^{\mathrm{b}, \mathrm{c}}$ \\
SOFA & $0[0-0]$ & $4[1-5.75]^{\mathrm{a}}$ & $7[5.5-11.0]^{\mathrm{b}, \mathrm{c}}$ \\
Upper gastrointestinal surgery/colorectal surgery & $9 / 41$ & $7 / 9$ & $13 / 24$ \\
\hline
\end{tabular}

Data are presented as frequencies or median and $25^{\text {th }}-75^{\text {th }}$ percentile values.

${ }^{a}$ statistically significant difference $(p<0.05)$ between septic patients without SA-AKI and control group.

${ }^{b}$ statistically significant difference $(\mathrm{p}<0.05)$ between septic patients with SA-AKI and control group.

${ }^{\mathrm{c}}$ statistically significant difference $(\mathrm{p}<0.05)$ between septic patients with SA-AKI and septic patients without SA-AKI; Mann-Whitney $\mathrm{U}$ test, Chi square test.

SA-AKI, sepsis-associated acute kidney injury; ASA, American Society of Anesthesiologists physical status; APACHE II, Acute Physiology and Chronic Health Evaluation II score; SOFA, Sequential Organ Failure Assessment score.

Table 2. Baseline laboratory parameters in patients with sepsis and the control group.

\begin{tabular}{lccc}
\hline & Control group & Sepsis without SA-AKI & Sepsis with SA-AKI \\
\hline Urea $(\mathrm{mmol} / \mathrm{L})$ & $4.9[3.6-6.7]$ & $7.2[5.4-11.0]^{\mathrm{a}}$ & $17.6[10.5-22.5]^{\mathrm{b}, \mathrm{c}}$ \\
Creatinine $(\mu \mathrm{mol} / \mathrm{L})$ & $74.0[56.7-92.0]$ & $74.4[56.0-90.1]$ & $168.1[124.7-233.9]^{\mathrm{b}, \mathrm{c}}$ \\
GFR $(\mathrm{ml} / \mathrm{min})$ & $90.5[67.5-15.8]$ & $90.5[49.6-116.7]$ & $32.8[21.2-53.5]^{\mathrm{bcc}}$ \\
Serum NGAL $(\mathrm{ng} / \mathrm{ml})$ & $100.3[91.2-111.9]$ & $109.5[85.4-173.4]$ & $184.3[112.7-209.6]^{\mathrm{b}, \mathrm{c}}$ \\
Urine NGAL $(\mathrm{ng} / \mathrm{ml})$ & $42.9[27.2-57.6]$ & $54.0[27.7-169.6]$ & $150.7[86.7-207.3]^{\mathrm{b}, \mathrm{c}}$ \\
Urine KIM-1 $(\mathrm{pg} / \mathrm{ml})$ & $800.0[730.0-1977.0]$ & $706.8[667.0-763.8]$ & $829.5[734.7-2018.0]^{\mathrm{c}}$ \\
MMP-9 $(\mathrm{ng} / \mathrm{ml})$ & $663.3[537.2-726.1]$ & $686.3[409.7-1358.6]$ & $533.0[370.7-884.9]$ \\
TIMP-1 $(\mathrm{ng} / \mathrm{ml})$ & $263.0[153.2-428.1]$ & $513.2[253.2-594.1]^{\mathrm{a}}$ & $577.2[505.8-1040.9]^{\mathrm{b}, \mathrm{c}}$ \\
MMP-9/TIMP-1 ratio & $2.47[1.59-3.61]$ & $2.25[1.15-2.76]$ & $0.8[0.5-0.2]^{\mathrm{b}, \mathrm{c}}$ \\
\hline
\end{tabular}

Data are presented as median and $25^{\text {th }}-75^{\text {th }}$ percentile values.

${ }^{a}$ statistically significant difference $(\mathrm{p}<0.05)$ between septic patients without SA-AKI and control group.

${ }^{b}$ statistically significant difference $(p<0.05)$ between septic patients with SA-AKI and control group.

${ }^{\mathrm{c}}$ statistically significant difference $(\mathrm{p}<0.05)$ between septic patients with SA-AKI and septic patients without SA-AKI; MannWhitney U test.

SA-AKI, sepsis-associated acute kidney injury, GFR, glomerular filtration rate; NGAL, neutrophile gelatinase-associated lipocalin; KIM-1, kidney injury molecule-1; MMP-9, matrix metalloproteinase-9; TIMP-1, tissue inhibitor of matrix metalloproteinase-1. 

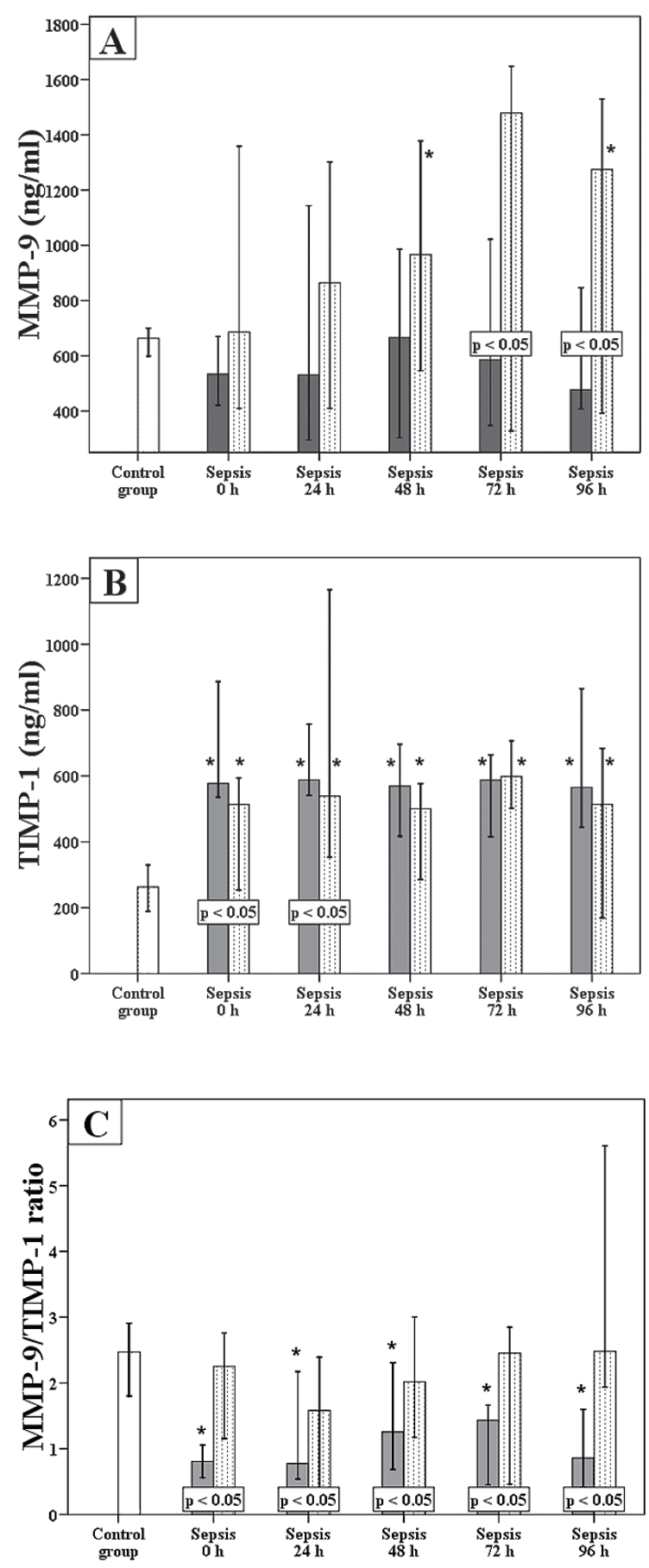

Fig. 1. Dynamics of MMP-9, TIMP-1, and MMP-9/TIMP-1 ratio.

The graphs show the time course of MMP-9 levels (A), TIMP-1 levels (B), and MMP-9/TIMP-1 ratio (C). Data are expressed as median and $95 \%$ confidence interval. White bars, control group; dotted bars, septic patients without SA-AKI; and shaded bars, septic patients with SA-AKI. $\mathrm{P}<0.05$ within a box indicates statistically significant difference between septic patients with and those without SA-AKI. ${ }^{*} p<0.05$, compared to control group (Mann-Whitney U test).

SA-AKI, sepsis-associated acute kidney injury; MMP-9, matrix metalloproteinase-9; TIMP-1, tissue inhibitor of matrix metalloproteinase- 1 . ing the admission to ICU. However, MMP-9 levels in septic patients without SA-AKI at $48 \mathrm{~h}$ and $96 \mathrm{~h}$ after admission to ICU were significantly higher than in the control group (Fig. 1A). As seen in Fig. 1B, the septic patients with SA-AKI had higher TIMP-1 levels compared to the septic patients without SA-AKI on the admission to ICU and $24 \mathrm{~h}$ later. Both septic patients with and without SA-AKI had, at all time points, higher levels of TIMP-1 compared to the control group. MMP-9/TIMP-1 ratio was significantly lower at all time points in the septic patients with SA-AKI relative to the septic patients without SA-AKI and the control group (Fig. 1C).

According to Friedman's repeated measures test, levels of MMP-9 and TIMP-1 and MMP-9/TIMP-1 ratio did not change significantly during the first $96 \mathrm{~h}$ of the ICU stay in the septic patients with or without SA-AKI ( $p>0.05)$.

Statistically significant correlation between MMP-9 and TIMP-1 was not observed (Table 3). MMP-9 and MMP-9/TIMP-1 ratio were negatively and TIMP-1 positively correlated with urea, creatinine, NGAL and SOFA scores. Also, a positive correlation was observed between TIMP-1 and KIM-1.

The ROC curves were constructed to evaluate MMP9, TIMP-1 and MMP-9/TIMP-1 ratio as possible diagnostic biomarkers of SA-AKI in patients with sepsis (Table 4). We found that TIMP-1 and MMP-9/TIMP-1 ratio, but not MMP-9, were good diagnostic biomarkers of SA-AKI, comparable to NGAL and KIM-1. As expected, combination of biomarkers increased their ability to discriminate between the septic patients with and without SA-AKI.

\section{Discussion}

In this study, the patients with SA-AKI had higher TIMP-1 levels and lower MMP-9 levels and MMP-9/TIMP ratio compared to the septic patients without SA-AKI and the control group. The levels of these biomarkers did not change significantly over time. MMP-9, TIMP-1 and MMP-9/TIMP-1 ratio correlated with disease severity scores, standard and novel biomarkers of AKI. TIMP-1 and MMP-9/TIMP-1 ratio were, unlike MMP-9, good diagnostic biomarkers of SA-AKI. Our results suggest that TIMP-1 and MMP-9/TIMP-1 ratio could serve as potential diagnostic biomarkers of SA-AKI in major abdominal surgery patients with sepsis.

The novelty of our study is the exploration of MMP-9 and TIMP-1 in SA-AKI in a specific population of major abdominal surgery patients with sepsis. This was unique for two reasons. First, even though animal models of SA-AKI repeatedly demonstrate elevated kidney expression of MMP-9 and TIMP-1 (Maitra et al. 2010; Teng et al. 2012), there has been only one clinical study to explore MMP-9 levels in AKI, but not SA-AKI per se (Han et al. 2008). Second, given that major abdominal surgery patients with sepsis are considered as high risk patients for developing AKI (Kheterpal et al. 2009), it is surprising that novel biomarkers of AKI have not yet been evaluated in this spe- 
Table 3. Correlation coefficients between tested parameters in patients with sepsis.

\begin{tabular}{lccc}
\hline \multicolumn{1}{c}{ Kendall tau-b coefficient } & MMP-9 & TIMP-1 & MMP-9/TIMP-1 ratio \\
\hline APACHE II & -0.099 & 0.107 & $-0.116^{*}$ \\
SOFA & $-0.149^{*}$ & $0.181^{*}$ & $-0.196^{*}$ \\
Serum Urea & $-0.154^{*}$ & 0.103 & $-0.167^{*}$ \\
Serum Creatinine & $-0.187^{*}$ & $0.153^{*}$ & $-0.234^{*}$ \\
GFR & $0.193^{*}$ & $-0.135^{*}$ & $0.237^{*}$ \\
Serum NGAL & -0.089 & $0.271^{*}$ & $-0.203^{*}$ \\
Urine NGAL & $-0.277^{*}$ & $0.197^{*}$ & $-0.338^{*}$ \\
Urine KIM-1 & -0.002 & $0.144^{*}$ & -0.104 \\
\hline
\end{tabular}

*statistically significant correlation $(\mathrm{p}<0.05)$; Kendal tau-b correlation analysis.

APACHE II, Acute Physiology and Chronic Health Evaluation II score; SOFA, Sequential Organ Failure Assessment score; GFR, glomerular filtration rate; NGAL, neutrophile gelatinase-associated lipocalin; KIM-1, kidney injury molecule-1; MMP-9, matrix metalloproteinase-9; TIMP-1, tissue inhibitor of matrix metalloproteinase-1.

Table 4. MMP-9, TIMP-1 and MMP-9/TIMP-1 ratio as diagnostic biomarkers of SA-AKI in patients with sepsis.

\begin{tabular}{lcc}
\hline & \multicolumn{1}{c}{ AUC-ROC $(95 \%$ C.I. $)$} \\
\cline { 2 - 3 } & On admission to ICU $(0 \mathrm{~h})$ & Mean $(0 \mathrm{~h}, 24 \mathrm{~h}, 48 \mathrm{~h}, 72 \mathrm{~h}, 96 \mathrm{~h})$ \\
\hline MMP-9 & $0.641(0.478-0.811)$ & $0.642(0.474-0.810)$ \\
TIMP-1 & $0.703(0.547-0.858)^{*}$ & $0.688(0.522-0.854)^{*}$ \\
MMP-9/TIMP-1 ratio & $0.740(0.580-0.901)^{*}$ & $0.691(0.532-0.850)^{*}$ \\
MMP-9 + TIMP-1 & $0.770(0.620-0.916)^{*}$ & $0.714(0.558-0.870)^{*}$ \\
uNGAL & $0.721(0.569-0.837)^{*}$ & $0.724(0.569-0.879)^{*}$ \\
uKIM-1 & $0.688(0.505-0.872)^{*}$ & $0.645(0.464-0.826)^{*}$ \\
SNGAL + uNGAL + uKIM-1 & $0.788(0.649-0.926)^{*}$ & $0.713(0.558-0.867)^{*}$ \\
\hline
\end{tabular}

Data are presented as AUC-ROC (95\% C.I.).

*statistically significant $(\mathrm{p}<0.05)$.

SA-AKI, sepsis-associated acute kidney injury; AUC-ROC, area under reciever operating characteristic curve; MMP-9, matrix metalloproteinase-9; TIMP-1, tissue inhibitor of matrix metalloproteinase-1; sNGAL, serum neutrophile gelatinase-associated lipocalin; uNGAL, urine neutrophile gelatinase-associated lipocalin; KIM-1, kidney injury molecule- 1 .

cific population.

We also introduced a unique control group consisting of patients without sepsis or AKI who match the septic patients by age, gender, comorbidities and a type of surgery. The previous studies on MMP-9 and TIMP-1 levels in sepsis compared septic patients to either healthy individuals (Lauhio et al. 2011; Muhl et al. 2011; Yazdan-Ashoori et al. 2011; Wang et al. 2014) or other critically ill patients (Martin et al. 2014) or they compared different subgroups of septic patients (Lorente et al. 2014) not taking into account any possible effects of surgery-induced inflammation (Helmersson-Karlqvist et al. 2012; Lindholm et al. 2015) or comorbidities on MMP-9 and TIMP-1 levels. Comorbidities present in patients with sepsis could alter levels of MMP-9 and TIMP-1 (Vandooren et al. 2013; Galliera et al. 2015) and are also considered as independent risk factors for AKI in the perioperative period (Kheterpal et al. 2009).

In our study, the patients with SA-AKI had lower lev- els of MMP-9 relative to the septic patients without SA-AKI. This result was unexpected for two reasons. First, animal studies on SA-AKI demonstrate increased MMP-9 kidney expression (Maitra et al. 2010; Teng et al. 2012). Until now, only one small clinical study explored MMP-9 in AKI to find elevated levels of urinary MMP-9 in pediatric patients with AKI, but not SA-AKI per se (Han et al. 2008). Second, consistently with previous reports (Wang et al. 2014; Dai et al. 2015), we found higher levels of NGAL in patients with SA-AKI. Considering that NGAL protects MMP-9 from degradation (Chakraborty et al. 2012), a negative correlation between NGAL and MMP-9, which was observed in our study, was unexpected (Wang et al. 2014). One could argue that other factors, like inhibition by TIMP-1 (Vandooren et al. 2013), influence MMP-9 activity more severely than does NGAL. Besides, NGAL, unlike KIM-1, is not elevated only due to SA-AKI but also due to inflammation (Martensson and Bellomo 2014). This is corroborated by our results, which imply absence of sig- 
nificant correlation between MMP-9 and KIM-1. Further clinical research on the association between NGAL and MMP-9 is needed.

We also found that the septic patients without SA-AKI had higher levels of MMP-9 compared to the control group which indicates that sepsis not complicated by SA-AKI, causes the increase in MMP-9 activity. In sepsis, both higher (Lauhio et al. 2011; Muhl et al. 2011; YazdanAshoori et al. 2011; Wang et al. 2014) and lower (Martin et al. 2014) levels of MMP-9 were found but none of these studies reported incidence of SA-AKI in the study population.

We observed higher levels of TIMP-1 in the septic patients with SA-AKI compared to the septic patients without SA-AKI as well as in the septic patients without SA-AKI compared to the control group. Previous experimental (Maitra et al. 2010; Teng et al. 2012) and clinical studies also report higher levels of TIMP-1 in sepsis (Lauhio et al. 2011; Muhl et al. 2011; Yazdan-Ashoori et al. 2011; Lorente et al. 2014) and SA-AKI (Wang et al. 2014).

Under normal conditions, a balance exists between MMP-9 and TIMP-1 and disease states are associated with an imbalance (Vandooren et al. 2013). Previous studies explored MMP-9/TIMP-1 (Muhl et al. 2011) and TIMP-1/ MMP-9 (Lorente et al. 2014) ratios in sepsis but not in SA-AKI. In our study, patients with SA-AKI had reduced MMP-9/TIMP-1 ratios at all time points compared to the septic patients without SA-AKI and the control group. Values of MMP-9/TIMP-1 ratio in the septic patients did not change significantly during the first $96 \mathrm{~h}$ in the ICU, which is supported by Muhl et al. (2011). Lorente et al. (2014) recognized the importance of MMP-9/TIMP-1 ratio as a predictive biomarker of the sepsis outcome.

Our study was the first to evaluate MMP-9 and TIMP-1 as diagnostic biomarkers of SA-AKI in surgical patients with sepsis. We found that TIMP-1 levels and MMP-9/TIMP-1 ratio were, unlike MMP-9, good diagnostic biomarkers of SA-AKI, comparable to NGAL and KIM1. Wang et al. (2014) also found TIMP-1 to be a good diagnostic marker of SA-AKI but in the emergency department settings. As expected, the combination of biomarkers showed greater diagnostic power for SA-AKI compared to individual biomarkers.

In conclusion, we report for the first time the association among MMP-9, TIMP-1 and SA-AKI. Major abdominal surgery patients with SA-AKI had higher levels of TIMP-1 and lower levels of MMP-9 and MMP-9/TIMP-1 ratio. TIMP-1 and MMP-9/TIMP-1 ratio could serve as potential diagnostic biomarkers of SA-AKI. Further evaluation of MMP- 9 and TIMP-1 in diagnosis and, possibly, prognosis of SA-AKI is necessary.

\section{Acknowledgments}

This study was partially supported by grants from Ministry of Education, Science and Technological Development, Republic of Serbia (Project No. 175035 and 175089).

\section{Conflict of Interest}

The authors declare no conflict of interest.

\section{References}

Alobaidi, R., Basu, R.K., Goldstein, S.L. \& Bagshaw, S.M. (2015) Sepsis-associated acute kidney injury. Semin. Nephrol., 35, $2-11$.

Berger, M.M., Marazzi, A., Freeman, J. \& Chiolero, R. (1992) Evaluation of the consistency of Acute Physiology and Chronic Health Evaluation (APACHE II) scoring in a surgical intensive care unit. Crit. Care Med., 20, 1681-1687.

Bone, R.C., Balk, R.A., Cerra, F.B., Dellinger, R.P., Fein, A.M., Knaus, W.A., Schein, R.M. \& Sibbald, W.J. (1992) Definitions for sepsis and organ failure and guidelines for the use of innovative therapies in sepsis. The ACCP/SCCM Consensus Conference Committee. American College of Chest Physicians/Society of Critical Care Medicine. Chest, 101, 16441655 .

Chakraborty, S., Kaur, S., Guha, S. \& Batra, S.K. (2012) The multifaceted roles of neutrophil gelatinase associated lipocalin (NGAL) in inflammation and cancer. Biochim. Biophys. Acta, 1826, 129-169.

Chertow, G.M., Burdick, E., Honour, M., Bonventre, J.V. \& Bates, D.W. (2005) Acute kidney injury, mortality, length of stay, and costs in hospitalized patients. J. Am. Soc. Nephrol., 16, 3365-3370.

Cullen, M.R., Jhanji, S., Pearse, R.M. \& Fitzgibbon, M.C. (2014) Neutrophil gelatinase-associated lipocalin and albuminuria as predictors of acute kidney injury in patients treated with goaldirected haemodynamic therapy after major abdominal surgery. Ann. Clin. Biochem., 51, 392-399.

Dai, X., Zeng, Z., Fu, C., Zhang, S., Cai, Y. \& Chen, Z. (2015) Diagnostic value of neutrophil gelatinase-associated lipocalin, cystatin $\mathrm{C}$, and soluble triggering receptor expressed on myeloid cells- 1 in critically ill patients with sepsis-associated acute kidney injury. Crit. Care, 19, 223.

Galliera, E., Tacchini, L. \& Corsi Romanelli, M.M. (2015) Matrix metalloproteinases as biomarkers of disease: updates and new insights. Clin. Chem. Lab. Med., 53, 349-355.

Han, W.K., Waikar, S.S., Johnson, A., Betensky, R.A., Dent, C.L., Devarajan, P. \& Bonventre, J.V. (2008) Urinary biomarkers in the early diagnosis of acute kidney injury. Kidney Int., 73, 863-869.

Helmersson-Karlqvist, J., Akerfeldt, T., Gunningberg, L., Swenne, C.L. \& Larsson, A. (2012) Serum MMP-9 and TIMP-1 concentrations and MMP-9 activity during surgery-induced inflammation in humans. Clin. Chem. Lab. Med., 50, 11151119.

Kheterpal, S., Tremper, K.K., Heung, M., Rosenberg, A.L., Englesbe, M., Shanks, A.M. \& Campbell, D.A. Jr. (2009) Development and validation of an acute kidney injury risk index for patients undergoing general surgery: results from a national data set. Anesthesiology, 110, 505-515.

Khwaja, A. (2012) KDIGO clinical practice guidelines for acute kidney injury. Nephron Clin. Pract., 120, c179-184.

Lauhio, A., Hastbacka, J., Pettila, V., Tervahartiala, T., Karlsson, S., Varpula, T., Varpula, M., Ruokonen, E., Sorsa, T. \& Kolho, E. (2011) Serum MMP-8, -9 and TIMP-1 in sepsis: high serum levels of MMP-8 and TIMP-1 are associated with fatal outcome in a multicentre, prospective cohort study. Hypothetical impact of tetracyclines. Pharmacol. Res., 64, 590-594.

Levey, A.S., Bosch, J.P., Lewis, J.B., Greene, T., Rogers, N. \& Roth, D. (1999) A more accurate method to estimate glomerular filtration rate from serum creatinine: a new prediction equation. Modification of Diet in Renal Disease Study Group. Ann. Intern. Med., 130, 461-470. 
Lindholm, E.E., Aune, E., Seljeflot, I., Otterstad, J.E. \& Kirkeboen, K.A. (2015) Biomarkers of inflammation in major vascular surgery: a prospective randomised trial. Acta Anaesthesiol. Scand., 59,773-787.

Little, J.P. (1995) Consistency of ASA grading. Anaesthesia, 50, 658-659.

Lorente, L., Martin, M.M., Sole-Violan, J., Blanquer, J., Labarta, L., Diaz, C., Borreguero-Leon, J.M., Orbe, J., Rodriguez, J.A., Jimenez, A. \& Paramo, J.A. (2014) Association of sepsisrelated mortality with early increase of TIMP-1/MMP-9 ratio. PLoS One, 9, e94318.

Maitra, S.R., Jacob, A., Zhou, M. \& Wang, P. (2010) Modulation of matrix metalloproteinase- 9 and tissue inhibitor of matrix metalloproteinase-1 in sepsis. Int. J. Clin. Exp. Med., 3, 180-185.

Martensson, J. \& Bellomo, R. (2014) The rise and fall of NGAL in acute kidney injury. Blood Purif., 37, 304-310.

Martin, G., Asensi, V., Montes, A.H., Collazos, J., Alvarez, V., Carton, J.A., Taboada, F. \& Valle-Garay, E. (2014) Role of plasma matrix-metalloproteases (MMPs) and their polymorphisms (SNPs) in sepsis development and outcome in ICU patients. Sci. Rep., 4, 5002.

Moreno, R., Vincent, J.L., Matos, R., Mendonca, A., Cantraine, F., Thijs, L., Takala, J., Sprung, C., Antonelli, M., Bruining, H. \& Willatts, S. (1999) The use of maximum SOFA score to quantify organ dysfunction/failure in intensive care. Results of a prospective, multicentre study. Working Group on Sepsis related Problems of the ESICM. Intensive Care Med., 25, 686-696.

Muhl, D., Nagy, B., Woth, G., Falusi, B., Bogar, L., Weber, G. \& Lantos, J. (2011) Dynamic changes of matrix metalloproteinases and their tissue inhibitors in severe sepsis. J. Crit. Care, 26, $550-555$.

Shao, X., Tian, L., Xu, W., Zhang, Z., Wang, C., Qi, C., Ni, Z. \& Mou, S. (2014) Diagnostic value of urinary kidney injury molecule 1 for acute kidney injury: a meta-analysis. PLoS
One, 9, e84131.

Shavit, L., Dolgoker, I., Ivgi, H., Assous, M. \& Slotki, I. (2011) Neutrophil gelatinase-associated lipocalin as a predictor of complications and mortality in patients undergoing noncardiac major surgery. Kidney Blood Press. Res., 34, 116-124.

Teixeira, C., Rosa, R., Rodrigues, N., Mendes, I., Peixoto, L., Dias, S., Melo, M.J., Pereira, M., Bicha Castelo, H. \& Lopes, J.A. (2014) Acute kidney injury after major abdominal surgery: a retrospective cohort analysis. Crit. Care Res. Pract., 2014, 132175.

Teng, L., Yu, M., Li, J.M., Tang, H., Yu, J., Mo, L.H., Jin, J. \& Liu, X.Z. (2012) Matrix metalloproteinase-9 as new biomarkers of severity in multiple organ dysfunction syndrome caused by trauma and infection. Mol. Cell. Biochem., 360, 271-277.

Tu, Y., Wang, H., Sun, R., Ni, Y., Ma, L., Xv, F., Hu, X., Jiang, L., Wu, A., Chen, X., Chen, M., Liu, J. \& Han, F. (2014) Urinary netrin-1 and KIM-1 as early biomarkers for septic acute kidney injury. Ren. Fail., 36, 1559-1563.

Vandooren, J., Van den Steen, P.E. \& Opdenakker, G. (2013) Biochemistry and molecular biology of gelatinase B or matrix metalloproteinase-9 (MMP-9): the next decade. Crit. Rev. Biochem. Mol. Biol., 48, 222-272.

Wang, M., Zhang, Q., Zhao, X., Dong, G. \& Li, C. (2014) Diagnostic and prognostic value of neutrophil gelatinase-associated lipocalin, matrix metalloproteinase-9, and tissue inhibitor of matrix metalloproteinases-1 for sepsis in the Emergency Department: an observational study. Crit. Care, 18, 634.

Yazdan-Ashoori, P., Liaw, P., Toltl, L., Webb, B., Kilmer, G., Carter, D.E. \& Fraser, D.D. (2011) Elevated plasma matrix metalloproteinases and their tissue inhibitors in patients with severe sepsis. J. Crit. Care, 26, 556-565.

Zhou, F., Luo, Q., Wang, L. \& Han, L. (2015) Diagnostic value of neutrophil gelatinase-associated lipocalin for early diagnosis of cardiac surgery-associated acute kidney injury: a metaanalysis. Eur. J. Cardiothorac. Surg., [Epub ahead of print]. 